\title{
TIMING OF GAMMA-RAY PULSARS
}

\author{
G.S.BISNOVATYI-KOGAN \\ Space Research Institute, Moscow. \\ Email: gkogan@mx.iki.rssi.ru
}

Exept Geminga there could be other gamma ray pulsars with no radioemission, which are exhibited in EGRET observations as ordinary point-like sources [6]. Determination of pulsations in a hard gamma ray source is complicated by rareness of arriving quanta $\delta t \gg P$, and their small total number. When the value of the period is known from other observations (radio or $X$-ray), timing analysis gives the possibility to find this periodicity also in a gamma region [1]. When there is no information about the period, it could be found from pure gamma data [7], but this should take enormous amount of computer time. Here we describe a method for investigation of timing of gamma pulsars, represented by periodical objects with rare pulses, which gives possibility to determine 7 parameters of a gamma pulsar: frequency $\nu$, its two derivatives $\dot{\nu}$ and $\ddot{\nu}$, angular coordinates $\alpha$ and $\delta$ of the source, absolute value $v_{<}$and direction of a velocity of a proper motion, characterized by an angle $\theta$, see details in [4].

For timing analysis all data must be represented in the same coordinate system, which as a rule is connected with a barycenter of the Solar system. The space probe is in the point $S$ with Cartesian coordinates $\left(x_{0}, y_{0}, z_{0}\right)$, and direction to a source is defined by a straight line with coordinate angles $\alpha$ and $\delta$. The time interval $\Delta T$, which must be added to the moment of each event in the point $S$ to obtain a corresponding barycenter moment, is determined as $\Delta T=\frac{1}{c}\left(x_{0} \cos \delta \cos \alpha+y_{0} \cos \delta \sin \alpha+z_{0} \sin \delta\right)$ For small errors in $\alpha$ and $\delta$ we may find corresponding small barycenter corrections $\delta T=\frac{\partial \Delta T}{\partial \alpha} d \alpha+\frac{\partial \Delta T}{\partial \delta} d \delta$. In order to estimate an input of these errors on timing characteristics let us compare phases of the arriving signal calculated from measurements $\phi^{\prime}$ (with errors) and in true barycenter time $\phi$, so that $\phi^{\prime}=\int_{0}^{t^{\prime}} \nu^{\prime} d t^{\prime}, \quad \phi=\int_{0}^{t} \nu d t$. Here $t^{\prime}$ and $\nu^{\prime}$ are found after barycenter corrections, containing errors. Times $t$ and $t^{\prime}$ correspond to the same event. so we may rewrite $\phi^{\prime}$ in true barycenter coordinates as $\phi^{\prime}=\int_{0}^{t}\left[1+\frac{d \delta T}{d t}\right] d t$. To check the influence of these errors on timing we have investigated an artificial set of data, representing a source with proper parameters $\nu_{0}=4 \mathrm{~s}^{-1}, \dot{\nu}_{0}=-2 \cdot 10^{-13} \mathrm{~s}^{-2}, \ddot{\nu}_{0}=3 \cdot 10^{-26} \mathrm{~s}^{-3}, \omega=1.991063802 \cdot 10^{-7} \mathrm{~s}^{-1}$. The source parameters are chosen to satisfy a relation $\nu_{0} \ddot{\nu}_{0} / \dot{\nu}_{0}^{2}=3$. The Rayleigh criterion of periodicity [5] was used. The error in coordinates was taken equal to 5 arc seconds in absolute value $\sqrt{d \alpha^{2}+d \delta^{2}}$ with different $\alpha$ and $\delta$. While the error in $\ddot{\nu}^{\prime}$ is almost linearly proportional to the error, we get that for an angular error larger then $10^{-3}$ arc sec direct determination of $\ddot{\nu}^{\prime}$ by criterion gives about $100 \%$ error. This may be a reason for a high calculated breaking index of Geminga $[8,3]$. For a clean set of data all seven parameters chosen for modeling were determined with a precision, limited only by computational grid. Situation is becoming much more controversial when we apply it to a real data existing for the moment.

In application to Geminga we used COS-B and EGRET data. In the case of COS-B we used from 5 observational shifts all quanta with energy $E>50 \mathrm{MeV}$ laying in the circle $r=12.5 \cdot E^{-0.16}$, where $E$ is measured in $\mathrm{MeV}$ and $r$ in degrees, total of 1883 quanta. The criterion gives a gently sloping maximum at velocity $v_{<}=0^{\prime \prime} .2-0^{\prime \prime} .3$ per year, direction $\theta=40^{\circ}-60^{\circ}$, initial coordinate offsets $d \alpha_{0}$ and $d \delta_{0}$ equal to $-2^{\prime \prime}$ for both $\alpha$ - and $\delta$-axes with uncertainty about $2^{\prime \prime}$ for both coordinates, and periodicity parameters in a agreement with [8]. The obtained motion of Geminga does not contradict to the motion of $\mathrm{G}^{\prime \prime}$ star [2].

In the case of EGRET we used from 9 observational shifts the standard selection of all the quanta of the energy $E>70 \mathrm{MeV}$ laying in the circle $r=5.85 \cdot(E / 100)^{-0.534}$, total of 6751 quanta. There 
is a gently sloping maximum in the criterion value at $\nu_{0}=4.2177501295 \mathrm{~Hz}, \dot{\nu}_{0}=-1.9532 \cdot 10^{-13}$ $\mathrm{Hz} \cdot \mathrm{s}^{-1}, \ddot{\nu}_{0}=(22 \pm 13) \cdot 10^{-26} \mathrm{~Hz} \cdot \mathrm{s}^{-2}, v_{<}=0^{\prime \prime} .3-0^{\prime \prime} .4$ per year, $\theta=40^{\circ}-60^{\circ}, d \alpha_{0}$ and $d \delta_{0}$ both lay in the interval $-2^{\prime \prime}$ to $0^{\prime \prime}$.

Coordinates and motion of Geminga obtained from timing are in a satisfactory correspondence with the motion of $G^{\prime \prime}$ star when separately COS-B or EGRET data are used. Combined data set are in much worse agreement, probably, because of systematic errors between the data of two probes, and possible nonmonotonity of the period between 1982 and 1988 years (pulsar glitch). The value of the second derivative $\ddot{\nu}_{0}$ does not coincide with the theoretical one because of its high sencitivity to coordinate errors, implied by nonperfect choice of quanta. New types of gamma ray telescopes based on very wide aperture $(\geq 2.5 \pi$ steradian) and higher threshold of a few hundred $\mathrm{MeV}$ [9] would permit to get higher angular resolution $(\sim 1 \mathrm{arc} \min )$, reducing influence of a background, get $\sim 100$ better statistics due to continuous monitoring of larger part of the sky in this region.

Angular coordinates could be obtained with a precision which is better then follows from gamma or X ray data, but worse then in optical or radio observations. Observations of radio pulsars have shown, that their optical and $\mathrm{X}$-ray luminosity is decreasing with time much more rapidly, that radio and hard gamma radiation. So at increasing sensitivity we expect a discovery of tens of new gammaray pulsars similar to Geminga, may be without or very faint X-ray and optical counterparts. For such objects method of timing of gamma-ray pulsars developed above could become very important.

\section{References}

1. Bignami G.F., Caraveo P.A. Nature, 357, 287, 1992.

2. Bignami G.F., Caraveo P.A., Mereghetti S. Nature, 361, 704, 1993.

3. Bisnovatyi-Kogan G.S., Posnov K.A. Nature, 366, 663, 1993.

4. Bisnovatyi-Kogan G.S., Repin S.V. Astron. Astrophys. Transactions, 1997 (submitted)

5. Brazier K.T.S. MNRAS, 268, 709, 1994.

6. Brazier K.T.S. et al. MNRAS, 281, 1033, 1996

7. Gurin L.S. et al. Astrophys. Space Sci., 147, 307, 1988.

8. Hermsen W. et al. IAU Circular No. 5541, June 10, 1992.

9. Leikov N.G., Bisnovatyi-Kogan G.S. Ap. J. Suppl., 92, 679, 1994. 